\title{
SAPHO syndrome: pathogenesis, clinical presentation, imaging, comorbidities and treatment: a review
}

\author{
Hanna Przepiera-Będzak, Marek Brzosko \\ Department of Rheumatology, Internal Medicine, Geriatrics and Clinical Immunology, Pomeranian Medical University, Szczecin, Poland \\ Adv Dermatol Allergol 2021; XXXVIII (6): 937-942 \\ DOI: https://doi.org/10.5114/ada.2020.97394
}

\begin{abstract}
Synovitis, acne, pustulosis, hyperostosis and osteitis (SAPHO) syndrome is a constellation of dermatological and osteoarticular symptoms. The pathogenesis of SAPHO is unknown, but infectious, genetic, immunological and environmental factors may play a role. SAPHO is classified along two different spectrums: pustulo-psoriatic hyperostotic spondyloarthritis and chronic recurrent multifocal osteomyelitis. The typical skin lesions are palmoplantar pustulosis and acne. The sign of arthritis is involvement of the anterior chest wall, most often the sternoclavicular joints. There are no standard treatment recommendations, but nonsteroidal anti-inflammatory drugs, sulfasalazine, methotrexate, antibiotics and biological drugs can be considered.
\end{abstract}

Key words: synovitis, acne, pustulosis, hyperostosis and osteitis (SAPHO), pathogenesis, clinical presentation, imaging, comorbidities, treatment.

\section{Introduction}

Synovitis, acne, pustulosis, hyperostosis and osteitis (SAPHO) syndrome is a disease characterised by dermatological and osteoarticular symptoms and was introduced by Chamot et al. [1] in 1987. The diagnostic criteria for SAPHO syndrome were developed by Kahn et al. [2] in 1994.

Some authors suggest that SAPHO syndrome could be considered as a type of spondyloarthritis (SpA) or a type of psoriatic arthritis (PsA), but others maintain that these should be considered as separate diseases [3, 4].

$\mathrm{SAPHO}$ is more common in women than in men. The disease is predominant in middle-aged women [1, 3-9].

SAPHO syndrome is classified along two different spectrums: pustulo-psoriatic hyperostotic spondyloarthritis (PPHS) and chronic recurrent multifocal osteomyelitis (CRMO) [10].

The incidence of SAPHO syndrome is unknown. Most data on this syndrome come from Western and Northern Europe and from China and Japan [1, 7-9, 11-13].

The results of our own published observations, conducted on the largest group of SAPHO patients in Poland, play a significant role in this report. Relying on our own experience, we draw attention to essential problems concerning clinical symptoms and diagnostics of SAPHO patients, which will allow for rapid diagnosis of the disease. In the treatment of SAHO we have paid attention to the possibility of using antibiotics and biological drugs.

\section{Pathogenesis}

The pathogenesis of SAPHO syndrome is unknown. Infectious, genetic, immunological and environmental factors may play a role in the development of the disease.

The role of Propionibacterium acnes antigen as a factor initiating the inflammatory process is considered, although it has been only occasionally found in bacterial cultures [14-16].

Up to $30 \%$ of patients with SAPHO syndrome have been shown to be associated with the presence of the HLA-B 27 gene [4, 7].

An association has been established between copy number variations of the following genes: CSF2RA, NOD2, MEGF6, and ADAM5 and the predisposition of SAPHO syndrome [17].

Immune system dysfunction has been described in patients with SAPHO syndrome. Li et al. [18] found elevation of serum IgG4 (a subclass of immunoglobulin G) in SAPHO patients, and it correlated with activity of the disease.

Address for correspondence: Hanna Przepiera-Będzak MD, PhD, Department of Rheumatology, Internal Medicine, Geriatrics and Clinical Immunology, Pomeranian Medical University, 1 Unii Lubelskiej St, 71-252 Szczecin, Poland, phone: +48 914253321 , fax: +48 914253344 , e-mail: hannapb@pum.edu.pl

Received: 3.05.2020, accepted: 12.06.2020. 
Some cytokines have been considered to play a role in the pathogenesis of SAPHO. Increased serum tumour necrosis factor $\alpha$ (TNF- $\alpha$ ), which plays a role in osteitis, has been observed in SAPHO patients with CRMO [19]. It has been shown that increased serum interleukin 6 (IL-6) correlated positively with C-reactive protein (CRP) and erythrocyte sedimentation ratio (ESR) in SAPHO patients [20-23]. In SAPHO patients with anterior chest wall lesions, increased serum interleukin 8 (IL-8) has been observed, which suggests that this cytokine is involved in bone and joint inflammation [24]. Przepiera-Będzak et al. [25] found that increased serum levels of interleukin 18 (IL-18), and decreased serum levels of endothelin-1 (ET-1) were associated with an increased risk of palmoplantar pustulosis (PPP), characteristic skin lesion in SAPHO [25, 26]. This suggests that IL-18 and ET-1, by their influence on vasculopathy and inflammation, may play a role in the pathogenesis of skin changes in SAPHO syndrome [24-26]. In another study, an increased serum level of interleukin 23 (IL-23) in SAPHO patients was shown, but it did not correlate with disease activity assessed by CRP or ESR [23]. No significant correlations were found between angiogenic cytokine levels and clinical presentation in SAPHO patients [27].

\section{Clinical presentation}

SAPHO syndrome is classified along two different spectrums (Table 1) [10]:

1) PPHS, which is the most common form of the disease occurring in adults, consists of a triad of symptoms:

- sternocostoclavicular hyperostosis with fibrous osteomyelitis and painful ossifying periostitis. Symptoms are usually unilateral and can lead to narrowing of the adjacent subclavian vein;

- spondyloarthropathy with the formation of ossifying lesions in the spine, leading to the formation of syndesmophytes or parasyndesmophytes;

- palmoplantar pustulosis (PPP), which may be accompanied by peripheral and sacroiliac arthritis.

The course of the PPHS is mild and chronic with periods of exacerbation and remission. Skin lesion-type PPP is accompanied by symptoms of spondyloarthritis with hyperostosis.

2) CRMO in adults, which until 1997 was only described in children, involves:

- anterior chest wall with the involvement of the sternum and adjacent joints (50\%);

- lamellar bones (the lamellar part of the body of the bone, especially the femur) and pelvis;

- axial skeleton, with inflammation of one or several vertebrae, or intervertebral disc and adjacent vertebral bodies (spondylodiscitis). Involvement of the jawbone results in swelling of the face.

Skin lesions associated with CRMO include: PPP, acne, psoriasis and acne inversa [10].
In the adult form, CRMO can relapse, but remissions may persist for several years. In the course of the disease, signs of recurrent osteitis and arthritis can be observed [28].

The course of the disease is often chronic and eventually self-healing $[3,4,6,7,10]$.

\section{Skin changes}

The typical skin lesions are PPP and severe acne (Table 1) $[1-4,7,9,11]$. The pattern of skin changes could be as follows: about $80 \%$ of patients show PPP, about $5 \%$ acne and 5\% no skin changes [7]. Characteristic skin changes can precede the onset of arthritis (25\%) and can occur simultaneously with joint changes (63.5\%) or a certain period of time after the onset of arthritis (5.8\%) [7].

PPP presents in the form of yellow, sterile pustules, which are located mostly on the skin of the palmar parts of the hands and plantar parts of the feet [1, 2, 5-7, 10]. These pustules undergo spontaneous cracking and peeling, leaving no trace on the skin, and recurrence of skin lesions is observed. Bacteriological examination shows pustule secretion in most cases to be sterile, but in individual cases Propionibacterium acnes was cultured from the bacteriological culture of the pustule secretions [14-16].

Skin changes may be preceded by or coexist with the upper respiratory tract infection (laryngitis, bronchitis, sinusitis, middle ear infection) or urinary tract infection. However, the infection is not considered to be the cause of PPP, but it can exacerbate its course. Exacerbation of skin and joint changes stimulated by chronic stress have been also reported [1-4, 7, 8].

Another characteristic skin change is acne fulminans or acne inversa (hidradenitis suppurativa) [29]. Usually, exacerbation of skin lesions is accompanied by exacerbation of joint symptoms [1-4, 7-9]. Single cases of other rare skin lesions in the course of SAPHO syndrome have been described in the literature, such as pyoderma gangrenosum, Sneddon-Wilkinson disease, and Sweet's syndrome [29].

Involvement of internal organs in the course of SAPHO syndrome is rare and mainly affects soft tissues around the affected bone. There may be swelling and fibrosis of these tissues. The following organ changes have also been reported: vasoconstriction (mainly subclavian vein), pleurisy and pericarditis, ascending aortitis and enteritis [4, 30].

\section{Joint symptoms}

The first sign of arthritis is involvement of the anterior chest wall, most often the sternoclavicular joints and the first sternocostal joints, which are the areas most involved in SAPHO in up to $96 \%$ of cases. Characteristic symptoms are skin redness, oedema, palpation pain and bone hypertrophy located in the anterior chest wall, 
most commonly in the sternoclavicular joint [1-10, 24]. Shoulder joint pain and swelling (symmetrical or asymmetrical) is observed in $36.5 \%$ of patients, and small-joint arthritis in $4.5-13.5 \%[7,11]$.

In cases with CRMO, long bone, vertebral and mandibular pain and swelling is observed [7, 10, 31].

Mandibular involvement presented as mandibular osteitis is a rare localisation of bone involvement in SAPHO syndrome and was first described by Kahn et al. [31]. Mandibular osteitis demonstrating bone sclerosis mainly involves the body of the mandible and is usually associated with a severe course of the disease [7, 31-33].

In a paediatric and adult population of patients with CRMO it was observed that sternal involvement was more frequent in adults, whereas clavicle and long bone involvement was more frequent in children [28].

\section{Laboratory findings}

There is no specific laboratory test characteristic for SAPHO. Assessment of the HLA-B 27 gene is positive in up to $30 \%$ of cases $[4,7]$. In laboratory tests, features of an active inflammatory process (accelerated ESR, elevated C-reactive protein) are observed [3, 4, 7, 9, 11, 19, 24].

Rheumatoid factor and anti-cyclic citrullinated peptide antibody (anti-CCP) is usually negative, although in a very small percentage of patients, it can be positive $[7,11,24]$.

In about 1.9-39\% of SAPHO patients, antinuclear antibodies are positive, mostly in those with the CRMO type $[7,11,13,19]$. Also, in a small percentage of patients, a result positive for anticardiolipin antibodies was observed $[7,34]$.

\section{Imaging}

In addition to clinical assessment, imaging studies are important in the diagnosis of SAPHO syndrome (Table 1).

In early stages of the disease, bone scintigraphy is useful, and approximately $85-95 \%$ of patients have an increased tracer intake in the front wall of the chest, indicating an inflammatory process of the skeleton $[3,7$, $12,35,36]$. Changes are unilateral or bilateral in the area of the sternoclavicular, costochondral and costosternal joints [7, 12, 36-39]. However, not all patients who reported pain in the sternoclavicular joints show scintigraphic changes in this area. It should also be remembered that the result of a scintigraphic examination in the diagnosis of skeletal inflammatory changes may be unspecific [7, 12, 35, 36, 39].

Routine radiographic images of bones and joints present abnormalities in up to $26 \%$ of patients, most frequently as subchondral sclerosis in association with periosteal new bone formation. Erosions are observed much less frequently, in only $12 \%$ of patients with peripheral arthritis, and are most often associated with sclerosis. Con-
Table 1. Clinical characteristics and treatment options of the synovitis acne pustulosis hyperostosis osteitis syndrome

\begin{tabular}{|c|c|}
\hline $\begin{array}{l}\text { Cutaneous } \\
\text { manifestations }\end{array}$ & $\begin{array}{l}\text { Palmoplantar pustulosis } \\
\text { Acne fulminans or acne inversa }\end{array}$ \\
\hline $\begin{array}{l}\text { Osteoarticular } \\
\text { symptoms location }\end{array}$ & $\begin{array}{l}\text { PPHS: } \\
\text { - Sternoclavicular joints } \\
\text { - First sternocostal joints } \\
\text { - Shoulder joint } \\
\text { - CRMO: } \\
\text { - Sternum and adjacent joints } \\
\text { - Mandible } \\
\text { - Lamellar bones } \\
\text { - Vertebrae }\end{array}$ \\
\hline Changes in imaging & $\begin{array}{l}\text { Bone scintigraphy: } \\
\text { - Increased tracer intake in the front wall } \\
\text { of the chest } \\
\text { - Computed tomography: } \\
\text { - Erosions and sclerotic changes with } \\
\text { new bone formation and hyperostosis } \\
\text { - Magnetic resonance imaging: } \\
\text { - Bone marrow oedema }\end{array}$ \\
\hline $\begin{array}{l}\text { The most common } \\
\text { comorbidities }\end{array}$ & $\begin{array}{l}\text { Autoimmune diseases: } \\
\text { - Hypothyroidism } \\
\text { - Diabetes } \\
\text { - Sjögren syndrome } \\
\text { - Antiphospholipid syndrome } \\
\text { - Depression } \\
\text { - Metabolic syndrome }\end{array}$ \\
\hline Treatment options & $\begin{array}{l}\text { NSAIDs } \\
\text { Antibiotics: } \\
\text { - Doxycycline } \\
\text { - Azithromycin } \\
\text { - DMARDs: } \\
\text { - MTX } \\
\text { - SSZ } \\
\text { - Bisphosphonates } \\
\text { - Biologics: } \\
\text { - TNF- } \alpha \text { blockers } \\
\text { - IL-23/IL-17 axis blockers } \\
\text { - Small-molecule inhibitor of } \\
\text { phosphodiesterase-4 }\end{array}$ \\
\hline
\end{tabular}

CRMO - chronic recurrent multifocal osteomyelitis, DMARDs - disease-mod ifying antirheumatic drugs, IL-23/IL-17-interleukin 23/interleukin 17, MTX - methotrexate, NSAIDs - nonsteroidal anti-inflammatory drugs, PPHS pustule-psoriatic hyperostotic spondyloarthritis, SSZ - sulfasalazine, TNF- $\alpha$ - tumor necrosis factor alpha.

ventional radiographs of the sternocostoclavicular region show subchondral sclerosis and erosions with associated enthesopathy. Changes are usually asymmetrical [3, 4, 7, 12]. In patients with a short duration of the disease, the radiological image may be normal [7, 35, 39-42].

On computed tomography (CT) examination, erosions and sclerotic changes with new bone formation and hyperostosis are observed in up to $45 \%$ of patients [9, 36, 38-40]. The ligamentous ossifications are usually delicate [9]. In addition, the CT scans are useful in differentiating changes in the course of SAPHO syndrome with other disorders such as neoplasm [10, 31]. 
Magnetic resonance imaging (MRI) is useful in cases with CRMO and presents as bone marrow oedema in mandible, vertebral or lamellar bone [7, 12, 13, 31]. In 89\% of cases, sacroiliitis is observed in MRI assessment [13].

\section{Diagnosis and differential diagnoses}

The diagnosis of SAPHO syndrome is based on fulfilling at least one of the criteria described by Kahn et al. [2], which include: 1) chronic recurrent multifocal osteomyelitis, usually sterile, spine may be involved, with or without skin lesions, 2) acute, subacute or chronic arthritis associated with any of the following: PPP, pustular psoriasis or severe acne, and 3) any sterile osteitis associated with any of the following: PPP, pustular psoriasis, psoriasis vulgaris or severe acne.

The differential diagnoses of SAPHO syndrome include infectious neoplasm and other bone abnormalities such as: infectious osteomyelitis, infectious spondylodiscitis, osteosarcoma, Ewing sarcoma, bony metastasis, Paget's disease, eosinophilic granuloma, osteoarthritis and osteonecrosis. The biopsy of bone lesions with the assessment of histopathological changes and bacterial cultures are helpful to exclude bone neoplasms or infectious osteomyelitis [10, 31].

\section{Comorbidities}

There is not much known about the comorbidities in the course of SAPHO (Table 1).

An increased prevalence of autoimmune diseases in patients with SAPHO syndrome has been reported [7, 19, 43]. In a study by Przepiera-Będzak et al. [7], the most common comorbidities were hypothyroidism and diabetes. One patient had Sjögren syndrome, and one antiphospholipid syndrome. This could confirm the suggestion that autoimmunity may play a role in the pathomechanism of SAPHO syndrome [7, 19, 34, 43].

In a study by Hayem et al. [4], inflammatory bowel disease, mostly Crohn's disease, was observed in 7.5\% of patients; this coincidence, on the other hand, is characteristic of spondyloarthritis.

SAPHO patients have higher BMI than healthy controls and $19.5 \%$ of them meet criteria for metabolic syndrome (MetS). Disease activity correlates positively with levels of total cholesterol (TC) and LDL-cholesterol (LDL-C), which are well-known markers of the risk of cardiovascular disease. This suggests an increased risk of cardiovascular disease in this group of patients. Increased triglyceride (TG) and LDL-C levels and an increased prevalence of MetS in SAPHO patients treated with methotrexate (MTX) could suggest that in SAPHO patients MTX treatment promotes or exacerbates MetS [44].

It was reported that patients with psoriasis and PsA have an increased risk of depression $[45,46]$. PrzepieraBędzak et al. [7] observed a high prevalence of depres- sion in SAPHO patients. Patients suffered from a cosmetic disability caused by active PPP, and skin changes negatively affected their interpersonal contacts. This could suggest that SAPHO patients have an increased risk of depression. Effective treatment of skin lesions would have a beneficial effect on their quality of life [7].

\section{Treatment}

As the cause of SAPHO is unknown, there are no standard recommendations for treatment (Table 1).

Usually, the first-line drugs are nonsteroidal antiinflammatory drugs (NSAIDs). These drugs could reduce the joint symptoms, but have no influence on skin changes. NSAIDs are effective in only some patients [3, 4, 7, 9].

As Propionibacterium acnes is considered to be an important trigger of SAPHO syndrome, antibiotics are also useful in its treatment. Antibiotics such as doxycycline or azithromycin showed a good response in patients with active skin changes [7, 14-16].

There are data presented that the use of diseasemodifying antirheumatic drugs (DMARDs), particularly MTX and sulfasalazine (SSZ), has been effective in some patients [4, 7, 9]. Przepiera-Będzak et al. [7] observed good response in some patients to SSZ treatment, but in others they had to switch to MTX. In patients with mandibular osteomyelitis, glucocorticosteroids (GCS) can be considered [31].

Bisphosphonates may lead to remission in up to $73 \%$ of patients with CRMO after failure of NSAID and GCS therapy, especially in patients with vertebral involvement [47]. The safety of their use has been discussed. To date, no cases of osteonecrosis of the jaw have been reported in paediatric CRMO patients. Oral alendronate has been shown to be effective in treating refractory SAPHOsyndrome-type CRMO with femoral involvement [48]. In a study of 11 patients (9 F; 2 M) on SAPHO-type CRMO with mandibular involvement, a single infusion of ibandronate (6 mg i.v.) was effective in reducing pain in 7 patients, and in 4 patients two infusions of ibandronate were administered, resulting in a reduction of clinical symptoms and changes on scintigraphic examination [49].

The use of biologics in SAPHO could be effective in patients not responding to conventional treatment. There are data of successful treatment with TNF- $\alpha$ blockers. Infliximab was most commonly used in patients with $\mathrm{SAPHO}$, mostly at a dose of $5 \mathrm{mg} / \mathrm{kg}$. Dramatic improvement in joint pain and swelling symptoms was observed; however, in some cases an exacerbation of the skin disease was present [50-54]. There are data on a small number of cases concerning etanercept, adalimumab and certolizumab pegol treatment in SAPHO patients. The treatment was well tolerated, and clinical improvement was observed concerning joints, skin symptoms and mandibular osteomyelitis [55-58]. There are extremely limited data suggesting that interleukin 1 (IL-1) inhibition 
with anakinra does not seem effective in controlling skin disease in SAPHO patients $[59,60]$. Data regarding the use of newer biologics targeting the IL-23/IL-17 axis in patients with SAPHO are limited. The effectiveness of ustekinumab or secukinumab range from $40 \%$ to $60 \%$ [61].

There are little data concerning apremilast, an orally available small-molecule inhibitor of phosphodiesterase-4, which has been used successfully in the treatment of psoriasis and psoriatic arthritis [62].

Although IL-6 inhibition appears ineffective in SpA, adult-onset CRMO cases successfully treated with tocilizumab have been reported [63].

The SAPHO syndrome is a rare but very interesting problem. Knowledge of its characteristic symptoms and diagnostic procedures may increase the frequency of its early diagnosis and prevent osteoarticular progression. In addition, co-operation between rheumatologists and dermatologists will improve the quality of life for SAPHO patients.

\section{Conflict of interest}

The authors declare no conflict of interest.

\section{References}

1. Chamot AM, Benhamou CL, Kahn MF, et al. Le syndrome acné pustulose hyperostose ostéite (SAPHO). Résultats d'une enquęte nationale. 85 observations. Rev Rhum Mal Osteoartic 1987; 54: 187-96.

2. Kahn MF, Kahn MA. The SAPHO syndrome. Baillieres Clin Rheumatol 1994; 8: 333-62.

3. Colina M, Govoni M, Orzincolo C, Trotta F. Clinical and radiological evolution of synovitis, acne, pulstulosis, hyperostosis and osteitis syndrome: a single center study of a cohort of 71 subjects. Arthritis Rheum 2009; 61: 813-21.

4. Hayem G, Bouchaud-Chabot A, Benali K, et al. SAPHO syndrome: a long-term follow - up study of 120 cases. Semin Arthritis Rheum 1999; 29: 159-71.

5. Przepiera-Będzak H, Brzosko I, Brzosko M. Skeletal changes during the course of pustulosis palmoplantaris - report of two cases. Pol Arch Med Wewn 2002; 107: 59-62.

6. Przepiera-Bedzak H, Brzosko I, Fliciński J, et al. SAPHO syndrome - clinical features. Pol Arch Med Wewn 2006; 116: $1172-7$.

7. Przepiera-Będzak H, Brzosko M. Clinical symptoms, imaging, and treatment of SAPHO syndrome: a single-center study of 52 cases. Pol Arch Med Wew 2018; 128: 396-9.

8. Aljuhani F, Tournadre A, Tatar Z, et al. The SAPHO syndrome: a single-center study of 41 adult patients. J Rheumatol 2015; 42: 329-34.

9. Li C, Zuo Y, Wang J, et al. Synovitis, acne, pustulosis, hyperostosis and osteitis syndrome: a single centre study of a cohort of 164 patients. Rheumatology (Oxford) 2016; 55: 1023-30.

10. Schilling F, Kessler St. Das SAPHO syndrome: klinisch-rheumatologische und radiologische differenzierung und klassifizierung eines krankengutes von 86 fällen. Z Rheumatol 2000; 59: 1-28.
11. Okuno H, Watanuki M, Kuwahara Y, et al. Clinical features and radiological findings of 67 patients with SAPHO syndrome. Mod Rheumatol 2018; 28: 703-8.

12. Cao Y, Li C, Yang Q, et al. Three patterns of osteoarticular involvement in SAPHO syndrome: a cluster analysis based on whole body bone scintigraphy of 157 patients. Rheumatology 2019; 58: 1047-55.

13. Cao Y, Li C, Xu W, et al. Spinal and sacroiliac involvement in SAPHO syndrome: a single center study of a cohort of 354 patients. Semin Arthritis Rheum 2019; 48: 990-6.

14. Assmann G, Kueck O, Kirchhoff T, et al. Efficacy of antibiotic therapy for SAPHO syndrome is lost after its discontinuation: an interventional study. Arthritis Res Ther 2009; 11: R140.

15. Govoni M, Colina M, Massara A, Trotta F. SAPHO syndrome and infections. Autoimmun Rev 2009; 8: 256-9.

16. Colina M, Trotta F. Antibiotics may be useful in the treatment of SAPHO syndrome. Mod Rheumatol 2014; 24: 697-8.

17. Guo C, Tian X, Hand F, et al. Copy number variation exploration of multiple genes in SAPHO syndrome. J Rheumatol 2019; pii: jrheum.181393. doi: 10.3899/jrheum.181393.

18. Li C, Xiang Y, Wu X, et al. Serum IgG4 elevation in SAPHO syndrome: does it unmask a disease activity marker? Clin Exp Rheumatol 2020; 38: 35-41.

19. Jansson A, Renner ED, Ramser J, et al. Classification of nonbacterial osteitis: retrospective study of clinical, immunological and genetic aspects in 89 patients. Rheumatology 2007; 46: 154-60.

20. Gratacós J, Collado A, Filella X, et al. Serum cytokines (IL-6, TNF-alpha, IL-1 beta and IFN-gamma) in ankylosing spondylitis: a close correlation between serum IL- 6 and disease activity and severity. Br J Rheumatol 1994; 33: 927-31.

21. Bal A, Unlu E, Bahar G, et al. Comparison of serum IL-1 beta, SIL-2R, IL-6, and TNF-alpha levels with disease activity parameters in ankylosing spondylitis. Clin Rheumatol 2007; 26: 211-5.

22. Alenius GM, Eriksson C, Rantapää Dahlqvist S. Interleukin-6 and soluble interleukin-2 receptor alpha-markers of inflammation in patients with psoriatic arthritis? Clin Exp Rheumatol 2009; 27: 120-3.

23. Przepiera-Będzak H, Fischer K, Brzosko M. Serum IL-6 and IL-23 levels and their correlation with angiogenic cytokines and disease activity in ankylosing spondylitis, psoriatic arthritis, and SAPHO syndrome. Mediators Inflamm 2015; 2015: 785705.

24. Hurtado-Nedelec M, Chollet-Martin S, Nicaise-Roland P, et al. Characterization of the immune response in the synovitis, acne, pustulosis, hyperostosis, osteitis (SAPHO) syndrome. Rheumatology 2008; 47: 1160-7.

25. Przepiera-Będzak H, Fischer K, Brzosko M. Serum interleukin-18, fetuin-A, soluble intercellular adhesion molecule-1, and endothelin-1 in ankylosing spondylitis, psoriatic arthritis, and SAPHO syndrome. Int J Mol Sci 2016; 17: 1255.

26. Przepiera-Będzak H, Fischer K, Brzosko M. Extra-articular symptoms in constellation with selected serum cytokines and disease activity in spondyloarthritis. Mediators Inflamm 2016; 2016: 7617954.

27. Przepiera-Będzak H, Fischer K, Brzosko M. Serum levels of angiogenic cytokines in psoriatic arthritis and SAPHO syndrome. Pol Arch Med Wewn 2013; 123: 297-302.

28. Skrabl-Baumgartner A, Singer P, Greimel T, et al. Chronic non-bacterial osteomyelitis: a comparative study between children and adults. Pediatr Rheumatol Online J 2019; 17: 49. 
29. Vekic DA, Woods J, Lin P, Cains GD. SAPHO syndrome associated with hidradenitis suppurativa and pyoderma gangrenosum successfully treated with adalimumab and methotrexate: a case report and review of the literature. Int J Dermatol 2018; 57: 10-8.

30. Legoupil N, Revelon G, Allain J, et al. Iliac vein thrombosis complicating SAPHO syndrome: MRI and histologic features of soft tissue lesions. Joint Bone Spine 2001; 68: 79-83.

31. Kahn MF, Hayem F, Hayem G, Grossin M. Is diffuse scleros ing osteomyelitis of the mandible part of the synovitis, acne, pustulosis, hyperostosis, osteitis (SAPHO) syndrome? Analysis of seven cases. Oral Surg Oral Med Oral Pathol 1994; 78 594-8.

32. Pottecher P, Loffroy R, Estivalet L, et al. SAPHO syndrome revealed by sclerosing mandibular osteomyelitis. Diagn Interv Imaging 2014; 95: 885-7.

33. Ornetti $P$, Pottecher P. Clinical images: refractory SAPHO syndrome involving the mandible. Arthritis Rheumatol 2014; 66: 1966.

34. Przepiera-Będzak H, Brzosko M. Antiphospholipid syndrome with anti $\beta 2$ glicoprotein-1 antibodies as the cause of recurrent tibial vein thrombosis in SAPHO syndrome. Acta Dermatovenerol Croat 2016; 24: 305-6.

35. Edlund E, Johnsson U, Lidgren L, et al. Palmoplantar pustulosis and sternocostoclavicular arthro-osteitis. Ann Rheum Dis 1988; 47: 809-15.

36. Salles M, Olive A, Perez-Andres R, et al. The SAPHO syndrome: a clinical and imaging study. Clin Rheumatol 2011; 30: 245-9.

37. Nitzsche EU. Nuclear medicine imaging for diagnosis of CRPS I. Handchir Mikrochir Plast Chir 2011; 43: 20-4.

38. Linke R, Kuwert T, Uder M, et al. Skeletal SPECT/CT of the peripheral extremities. AJR Am J Roentgenol 2010; 194: 329-35.

39. Przepiera-Będzak H, Cyryłowski L, Brzosko I, et al. Diagnostyka obrazowa stawów mostkowo-obojczykowych u chorych na zespół SAPHO (ang. The imaging of sterno-clavicular joints in SAPHO syndrome). Post Nauk Med 2012; 25: 89-94.

40. Van Doornum S, Barraclough D, McColl G, Wicks I. SAPHO: rare or just not recognized? Semin Arthritis Rheum 2000; 30: 70-7.

41. Sonozaki $H$, Mitsui $H$, Miyanaga $Y$, et al. Clinical features of 53 cases with pustulotic arthro-osteitis. Ann Rheum Dis 1981 40: 547-53.

42. Maugars Y, Berthelot JM, Ducloux JM, Prost A. SAPHO syndrome: a followup study of 19 cases with special emphasis on enthesis involvement. J Rheumatol 1995; 22: 2135-41.

43. Valkema PA, Luymes $\mathrm{CH}$, Witteveen JE, et al. High prevalence of autoimmune disease in the rare inflammatory bone disorder sternocostoclavicular hyperostosis: survey of a Dutch cohort. Orphanet J Rare Dis 2017; 12: 20.

44. Przepiera-Będzak H, Fischer K, Brzosko M. Serum interleukin-23 protects, whereas methotrexate treatment stimulates selected components of the metabolic syndrome in patients with SAPHO syndrome. Arch Med Sci DOI:10.5114/ aoms.2018.76953.

45. Wu JJ, Penfold RB, Primatesta P, et al. The risk of depression, suicidal ideation and suicide attempt in patients with psoriasis, psoriatic arthritis or ankylosing spondylitis. J Eur Acad Dermatol Venereol 2017; 31: 1168-75.

46. Lewinson RT, Vallerand IA, Lowerison MW, et al. Depression is associated with an increased risk of psoriatic arthritis among patients with psoriasis: a population-based study. J Invest Dermatol 2017; 137: 828-35.
47. Taddio A, Ferrara G, Insalaco A, et al. Dealing with chronic non-bacterial osteomyelitis: a practical approach. Pediatr Rheumatol Online J 2017; 15: 87.

48. Ichikawa J, Sato E, Haro H, et al. Successful treatment of SAPHO syndrome with an oral bisphosphonate. Rheumatol Int 2009; 29: 713-5.

49. Otto S, Troeltzsch M, Burian E, et al. Ibandronate treatment of diffuse sclerosing osteomyelitis of the mandible: pain relief and insight into pathogenesis. J Craniomaxillofac Surg 2015; 43: 1837-42.

50. Massara A, Cavazzini PL, Trotta F. In SAPHO syndrome antiTNF-alpha therapy may induce persistent amelioration of osteoarticular complaints, but may exacerbate cutaneous manifestations. Rheumatology 2006; 45: 730-3.

51. Ben Abdelghani K, Dran DG, Gottenberg JE, et al. Tumor necrosis factor-alpha blockers in SAPHO syndrome. J Rheumatol 2010; 37: 1699-704.

52. Burgemeister LT, Baeten DLP, Tas SW. Biologics for rare inflammatory diseases: TNF blockade in the SAPHO syndrome. Neth J Med 2012; 70: 444-9.

53. Gupta AK, Skinner AR. A review of the use of infliximab to manage cutaneous dermatoses. J Cutan Med Surg 2004; 8: 77-89.

54. Olivieri I, Padula A, Ciancio G, et al. Successful treatment of SAPHO syndrome with infliximab: report of two cases. Ann Rheum Dis 2002; 61: 375-6.

55. Zhang L, Gao Z. Etanercept in the treatment of refractory SAPHO syndrome. Am J Clin Exp Immunol 2016; 5: 62-6.

56. Wagner AD, Andresen J, Jendro MC, et al. Sustained response to tumor necrosis factor alpha-blocking agents in two patients with SAPHO syndrome. Arthritis Rheum 2002; 46: 1965-8.

57. Marí A, Morla A, Melero M, et al. Diffuse sclerosing osteomyelitis (DSO) of the mandible in SAPHO syndrome: a novel approach with anti-TNF therapy. Systematic review. J Craniomaxillofac Surg 2014; 42: 1990-6.

58. Kamata Y, Minota S. Successful treatment of a patient with SAPHO syndrome with certolizumab pegol. Rheumatol Int 2015; 35: 1607-8.

59. Colina M, Pizzirani C, Khodeir M, et al. Dysregulation of P2X7 receptor-inflammasome axis in SAPHO syndrome: successful treatment with anakinra. Rheumatology 2010; 49: 1416-8.

60. Wendling D, Prati C, Aubin F. Anakinra treatment of SAPHO syndrome: short-term results of an open study. Ann Rheum Dis 2012; 71: 1098-100.

61. Wendling D, Aubin F, Verhoeven F, Prati C. IL-23/Th17 targeted therapies in SAPHO syndrome. A case series. Joint Bone Spine 2017; 84: 733-5.

62. Adamo S, Nilsson J, Krebs A, et al. Successful treatment of SAPHO syndrome with apremilast. Br J Dermatol 2018; 179: 959-62.

63. Sato H, Wada Y, Hasegawa E, et al. Adult-onset chronic recurrent multifocal osteomyelitis with high intensity of muscles detected by magnetic resonance imaging, successfully controlled with tocilizumab. Intern Med 2017; 56: 2353-60. 